\title{
Effects of cataract surgery and intra-ocular lens implantation on visual function and quality of life in age-related cataract patients: a systematic review protocol
}

\author{
Shalu Jain ${ }^{1 *}$, Kavitha Rajshekar ${ }^{1}$, Anjana Aggarwal ${ }^{1}$, Akshay Chauhan ${ }^{1}$ and Vijay Kumar Gauba ${ }^{1,2}$
}

\begin{abstract}
Background: Cataract is the leading cause of blindness and low vision worldwide. Presently, cataract surgery is the only treatment for cataract and is very effective in restoring sight. In cataract surgery, the natural lens of the eye that becomes clouded is removed and replaced with an artificial intraocular lens. There are multiple techniques for removal of lens as well as many types of intraocular lenses available for implantation. For this reason, it becomes imperative to monitor the impact of different surgical techniques and different intraocular lenses on health-related quality of life (HRQOL) of the patients. This systematic review aims to evaluate HRQoL evidences on effects of different types of cataract surgeries and intraocular lenses on visual function and quality of life in age-related cataract patients.

Method: Databases like Cochrane, EMBASE, SCOPUS, NHS Economic Evaluation Database (NHS EED), Health Technology Assessment (HTA) database, MEDLINE, ClinicalTrials.gov, Current Controlled Trials and World Health Organization International Clinical Trials Registry Platform (WHO ICTRP) will be searched systematically. Two reviewers will independently screen studies using predefined inclusion and exclusion criteria along with the extraction of data, and assessment of methodological quality using a standard checklist.

Discussion: This systematic review will help in understanding how different types of cataract surgeries and intraocular lenses make a difference on quality of life of age-related cataract patients in terms of visual function and health-related quality of life. As the review attempts to bring together all the cataract-related HRQoL evidences pertaining to different cataract surgical techniques, different intraocular lenses and cataractrelated complications, it will also identify gaps in evidence.
\end{abstract}

Systematic review registration: PROSPERO CRD42018092377

Keywords: Cataract, Health-related quality of life, Visual function, Health technology assessment, Economic evaluations, Cost effectiveness

\footnotetext{
* Correspondence: shaluisjain@gmail.com

${ }^{1}$ Health Technology Assessment in India, Department of Health Research,

Ministry of Health and Family Welfare, Indian Red Cross Society Building, 1,

Red Cross Road, New Delhi 110001, India

Full list of author information is available at the end of the article
}

(c) The Author(s). 2019 Open Access This article is distributed under the terms of the Creative Commons Attribution 4.0 International License (http://creativecommons.org/licenses/by/4.0/), which permits unrestricted use, distribution, and reproduction in any medium, provided you give appropriate credit to the original author(s) and the source, provide a link to the Creative Commons license, and indicate if changes were made. The Creative Commons Public Domain Dedication waiver (http://creativecommons.org/publicdomain/zero/1.0/) applies to the data made available in this article, unless otherwise stated. 


\section{Background}

Health Technology Assessment (HTA) is an internationally accepted tool to inform decision making for better management of existing resources for Universal Health Coverage (UHC) [1-3]. Health Technology Assessment in India (HTAIn) has been institutionalised in January 2017 under the Department of Health Research (DHR), Ministry of Health and Family Welfare (MoHFW) by the Government of India to facilitate the process of transparent and evidence-based decision-making for improved healthcare delivery [4]. HTAIn is entrusted with the responsibility to analyse evidences related to cost-effectiveness, clinical-effectiveness and equity issues regarding the deployment of health technologies like new medicines, devices and health programmes by means of HTA studies, which will in turn help in the efficient use of a limited health budget and provide people access to quality healthcare at minimum costs $[5,6]$.

The first HTA topic selected for study at HTAIn secretariat was 'Health Technology Assessment of intraocular lenses for treatment of age-related cataracts'. The request for this topic came from Rashtriya Swasthya Bima Yojana (RSBY), a Government health insurance scheme that has now been subsumed by the National Health Protection Scheme (NHPS) under Ayushman Bharat Mission [7, 8]. Under this HTA study, five individual literature reviews were conducted to gather the evidences namely on clinical effectiveness, cost effectiveness, health-related quality of life (HRQoL), costing and equity. The present study (systematic literature review on HRQoL) is a part of the HTA study on 'Health Technology Assessment of intraocular lenses for treatment of age-related cataracts'.

Cataract is a condition in which the lens of the eye becomes clouded preventing clear vision [9]. Cataract is the leading cause of blindness (51\%) and low vision (33\%) worldwide [10]. When vision $<20 / 200$ in the better eye on presentation is defined as blindness, it has also been reported that cataract is responsible for 50$80 \%$ of the bilaterally blind in the country [11-16].

Presently, cataract surgery is the only treatment for cataract, with high success rates, in restoring sight. The opaque lens of the eye is removed and replaced by an artificial intraocular lens [17, 18]. Cataract surgery as such is one of the most cost-effective interventions though cost and clinical effectiveness of different surgical techniques and intraocular lenses (IOLs) vary a lot [19]. In clinical decision-making, interventions are being primarily assessed based on efficacy and safety. However, it is also important to monitor the impact that treatments have on utility, i.e. health-related quality of life (HRQoL) using validated instruments [20, 21]. Utility is a measure of health preference anchored around a value of ' 1 ' for perfect health and ' 0 ' for dead that is used in calculations of quality-adjusted life years (QALY) [22].

This systematic review aims to evaluate health-related quality of life evidences on effects of different cataract surgeries and intraocular lens implantation on visual function and quality of life in age-related cataract patients. This review also attempts to bring together all the cataract-related HRQoL evidences pertaining to different cataract surgical techniques, different intraocular lenses and cataract-related complications.

\section{Methods}

This protocol has been registered a priori in PROSPERO (\#CRD42018092377) [23] and follows the Preferred Reporting Items for Systematic Reviews and Meta-Analyses (PRISMA) guidelines as included in Additional file 1.

\section{Review question}

What are the changes in quality of life and visual function in age-related cataract patients after undergoing a particular type of cataract surgery and intraocular lens implantation?

\section{Search strategy and information sources}

Strategies will be designed to identify all relevant studies for HRQoL among age-related cataract patients. We will search the bibliographic electronic databases like the Cochrane Library including the Cochrane Database of Systematic Reviews (CDSR), Cochrane Central Register of Controlled Trials (CENTRAL), EMBASE, SCOPUS, the NHS Economic Evaluation Database (NHS EED), Health Technology Assessment database, MEDLINE, ClinicalTrials.gov, Current Controlled Trials and World Health Organization International Clinical Trials Registry Platform (WHO ICTRP) systematically. For each database, we will use words and expressions from controlled vocabulary (MESH, EMTREE, and others) and free text searching. There will be no language or date restrictions for the literature search. Results will be managed using Covidence online software to facilitate automatic and manual removal of duplicate records, study screening and selection and record keeping. An example search strategy for EMBASE is given as Additional file 2.

\section{Criteria for considering studies for this review}

The studies will be included based on the following criteria:

\section{Inclusion criteria}

- Population: adult patients with age-related cataracts without any other ocular comorbidity 
- Interventions: phacoemulsification, small-incision cataract surgery (SICS), extracapsular cataract extraction (ECCE), intracapsular cataract extraction (ICCE), rigid lens, foldable lens, monofocal lens, multifocal lens

- Comparators: The comparators for the study would be as given below:

- Surgery: ICCE, ECCE, SICS, phacoemulsification.

- Lenses: rigid IOL, foldable IOL, monofocal IOL, multifocal IOL

- Type of cataract: unilateral cataract, bilateral cataract

- Outcomes: generic quality of life (QoL), HRQoL, vision-related quality of life (VRQoL), visual function (VF), cataract surgery-related complications

- Study design: systematic reviews, meta-analysis, randomised controlled trials (RCTs), original observational studies, case-control studies, cohort studies, cost-effectiveness studies

\section{Exclusion criteria}

- IOLs for non-age-related cataracts like congenital or paediatric cases

- Studies reporting insufficient data for analysis

- Studies evaluating the association between QoL and causes of visual impairment which are unrelated to cataract

- Studies validating the test questionnaire or construct validation studies

- Other eye disorders/diseases along with cataract

- Narrative review articles, study protocols, opinions, abstracts

Table 1 Preliminary data extraction table to be used for inclusion/exclusion of studies

\begin{tabular}{l}
\hline Serial No. \\
Study ID \\
Title of study \\
Published in year \\
Study design \\
Patient population \\
Intervention \\
Comparator (If any) \\
Outcome measures (e.g. visual acuity, QoL, VF) \\
Instruments used \\
Included/excluded \\
Reason for exclusion
\end{tabular}

Table 2 Data extraction table to be used for included studies

Category (study is related to surgical techniques, intraocular lenses, surgical complications or any other aspect of cataract)

Study ID

Title of the study

Year of publication

Country where the work is done

Aim of the study

Study settings

Study design

Randomisation method (If RCT ${ }^{a}$ )

Preference-based measures (generic/disease specific)

Which instrument is used

Sample size

Age criteria for patient recruitment

Patient recruitment details

Follow up details

Tariff details

Modelling details

Statistical tests used

Cost-effectiveness analysis (e.g, ICER ${ }^{\beta}, N H B^{\prime}$ )

Average age of patients

Visual function scores

Pre-surgery

Post-Surgery

Quality of life scores

Pre-surgery

Post-surgery

Results

Conclusion

Reviewer's remarks

${ }^{a}$ Randomised Controlled trials, $\beta$ - Incremental cost effectiveness ratio, $\gamma$ - Net Heath Benefit

$\beta$ Incremental cost-effectiveness ratio

${ }^{\gamma}$ Net heath benefit

\section{Year of publication and language}

Eligibility will not be restricted by year nor by language of publication.

\section{Inclusion screening process}

Studies will be selected for inclusion through a two-stage process. The first stage will be to screen the literature search results (titles and, if present, abstracts) identified by the search strategy to identify all citations that potentially meet the inclusion/exclusion criteria detailed above. The second stage will be a 'preliminary' data extraction to aid in the study selection process. Full manuscripts of selected citations that appeared potentially relevant will be obtained and preliminary data extraction will be done in order to ensure that all included studies have sufficient data pertaining to health-related generic 
Table 3 Quality assessment checklist used for included studies (modified from Ara et al.) [24]

\begin{tabular}{|c|c|}
\hline Criteria & Consideration \\
\hline \multicolumn{2}{|l|}{ Criteria 1. Relevance of the study } \\
\hline Relevant population & $\begin{array}{l}\text { How closely do the patient characteristics in the study match to the patient population we have described in } \\
\text { our inclusion criteria (PICO) }\end{array}$ \\
\hline Relevant health states & $\begin{array}{l}\text { Timing of data collection, e.g. pre-surgery, post-surgery, follow-up intervals. The use of any medications that is } \\
\text { likely to have an independent effect on HSUVs (either detrimental or beneficial) }\end{array}$ \\
\hline \multicolumn{2}{|l|}{ Criteria 2. Quality assessment } \\
\hline Sample size & This was not considered as exclusion criteria, but the precision of the estimate was assessed \\
\hline $\begin{array}{l}\text { Response rates to the measure } \\
\text { used }\end{array}$ & $\begin{array}{l}\text { Are response rates reported and if so, are the rates likely to be a threat to the validity of the estimated HSUVs } \\
\text { for the health states? }\end{array}$ \\
\hline Loss to follow-up & How large is the loss to follow-up and are these likely to threaten the validity of the estimates? \\
\hline Missing data & $\begin{array}{l}\text { What are the levels of missing data and how are they dealt with? } \\
\text { Are there details on the causes of the missing data? } \\
\text { Again, could this threaten the validity of the estimates? }\end{array}$ \\
\hline \multicolumn{2}{|c|}{ Criteria 3. Utility values are measured and valued appropriately } \\
\hline $\begin{array}{l}\text { Appropriate use of valuation } \\
\text { method }\end{array}$ & $\begin{array}{l}\text { If valuation methods are used (TTO, SG, DCE, VAS) they are used appropriately? } \\
\text { Does the valuation method provide preference based values anchored at } 1 \text { as equivalent to full health and } 0 \\
\text { as equivalent to dead? } \\
\text { Are adequate details of the valuation method provided to allow judgement on appropriateness? }\end{array}$ \\
\hline Appropriate use of GPMB & $\begin{array}{l}\text { Are adequate details of the PBM method provided (e.g. details given on the version used, the social tariff } \\
\text { applied) } \\
\text { Was the GPBM delivered as intended? (e.g. wording and response options not changed) } \\
\text { Is the measure used for the group it was intended (e.g. is an adult GPBM being used for children? Is EQ-5D-Y } \\
\text { used with the adult tariff?) }\end{array}$ \\
\hline $\begin{array}{l}\text { Appropriate health-state } \\
\text { description (vignette) }\end{array}$ & $\begin{array}{l}\text { If a health state is valued using a vignette, can the accuracy of the vignette be established? e.g. the process by } \\
\text { which it was derived is described }\end{array}$ \\
\hline
\end{tabular}

quality of life and/or vision-related quality of life (Table 1).

All studies will be assessed by two reviewers and checked independently by a third reviewer before taking a final decision. At each stage, any disagreements will be resolved by discussion.

\section{Data extraction process}

Two authors will extract data using a standardised data extraction form (Table 2) in Microsoft Excel, and the results will be compared for differences. Discrepancies in the extracted data will be resolved by discussion, with involvement of a third reviewer if necessary.

\section{Critical appraisal strategy}

The quality of included studies will be assessed by using a modified checklist recommended by Ara et al. [24] and insufficient detail in the primary studies will be noted under limitations. Three main criteria will be considered for quality assessment (Table 3). The fourth criteria from Ara et al. [24], which is 'In line with reimbursement agency requirements' will not be considered as the main aim of this study will be to systematically review cataract-related HRQoL evidences, and we do not intend to use the health state utility values (HSUVs) for a particular reimbursement agency here. Two authors will apply the criteria and it will be checked for differences with any disagreements resolved by consensus and involving a third reviewer wherever necessary.

\section{Method of data synthesis}

Studies finally selected for inclusion in the review will be classified into different categories based on the type of cataract surgery and/or intraocular lens it involves and then each category will be classified into the subcategories based on the type of QoL instrument used in the study. Data will be separately analysed for categories and subcategories accordingly.

We will pool the data for each category by performing a meta-analysis if we get enough studies with comparable outcome measures and with similarities in terms of study design, population, instruments used for measuring the health states, value sets used for assigning utility weights and reporting results. A fixed effects meta-analysis will be applied to obtain the pooled effect size with $95 \%$ confidence interval (CI) or else a random effects meta-analysis would be performed (heterogeneous, $\tau 2>0$ ). The heterogeneity level will be investigated by using $I^{2}$ index. An $I^{2}$ value of more than $75 \%$ will be considered as an indication of significant heterogeneity. If there is evidence for substantial heterogeneity or inconsistency, we will not pool the results. In 
circumstances where pooling of studies will be deemed inappropriate, we will only provide a qualitative discussion of the findings with tables of findings and a narrative description.

\section{Discussion}

Literature search on cataract-related HRQoL evidences results in a huge number of studies when generic terms for HRQoL are used. Cataract-related studies reporting HRQoL are diverse in terms of study design, type of preference-based measure used (disease-specific or generic measures) and type of instruments used (EQ 5D, Short Form 36, Visual Function Questionnaires like VF11 and VFQ-14, etc.) as there is no set pattern for reporting HRQoL. The diversity of studies is seen as some compare HRQoL between different types of intraocular lenses (e.g. comparison between monofocal and multifocal lenses) where others compare it between different types of cataract surgical techniques (e.g. comparison between phacoemulsification and small manual incision surgery). Still, others measure the HRQoL of cataract patients before and after surgery without mentioning much the details of type of surgical technique and IOLs used. Against this backdrop, the current study will be a broad review to systematically explore, critically appraise and provide insight to the effects of different types of cataract surgeries and intraocular lens implantations on visual function and quality of life in age-related cataract patients. If adequate evidence is available, this review may provide requisite data for decision modelling for economic evaluation studies. The review will also help in identifying gaps in availability of data that will help planning future modelling studies. As the review attempts to bring together all the cataract-related HRQoL evidences pertaining to different cataract surgical techniques, different intraocular lenses and cataract-related complications, it will also identify gaps in evidences and areas where future research is required.

\section{Additional files}

Additional file 1: PRISMA-P 2015 Checklist. (DOCX 30 kb)

Additional file 2: An example search strategy for EMBASE. (DOCX $19 \mathrm{~kb}$ )

\footnotetext{
Abbreviations

CDSR: Cochrane Database of Systematic Reviews; CENTRAL: Cochrane Central Register of Controlled Trials; DCE: Discrete Choice Experiment;

DHR: Department of Health Research; ECCE: Extracapsular cataract extraction; GPBM: Generic preference-based measures; HRQoL: Health-related quality of life; HSUV: Health state utility values; HTA: Health Technology Assessment; HTAln: Health Technology Assessment in India; ICCE: Intracapsular cataract extraction; ICER: Incremental cost-effectiveness ratio; IOL: Intraocular lens; MESH: Medical Subject Headings; NHB: Net health benefit; NHPS: National Health Protection Scheme; NHS EED: National Health Service Economic Evaluation Database; PBM: Preference-based measures; PICO: Population, Intervention, Comparator, Outcome; QALY: Quality-adjusted life years; QoL: Quality of life; RCT: Randomised controlled trial; RSBY: Rashtriya
}

Swasthya Bima Yojana; SG: Standard gamble; SICS: Small-incision cataract surgery; TTO: Time trade-off; UHC: Universal Health Coverage; VAS: Visual analogue scale; VF: Visual function; VRQoL: Vision-related quality of life; WHO ICTRP: World Health Organization International Clinical Trials Registry Platform

\section{Acknowledgements \\ This project is sponsored by the Department of Health Research and has an overall control of the design, conduct, analysis, interpretation and dissemination of the systematic review. The authors would like to thank Professor John E Brazier for helping with the data extraction form and critical appraisal methods. The authors would like to thank Dr. Nishant Jaiswal, Consultant, Evidence-Based Health Informatics, RRC North, Department of Telemedicine, PGIMER and Ms. Pranita Pradhan from ICMR Centre for Capacity building in Evidence-Based Medicine for North East Region, Department of Paediatrics, PGIMER, Chandigarh for helping in electronic searches of databases.}

\section{Authors' contributions}

SJ is the guarantor of the review. SJ, AA, AC and KR were involved in the design of the protocol and systematic review. SJ conducted the scoping searches. SJ, AA and AC piloted the inclusion/exclusion form. SJ and AC piloted the data extraction form. AA will be the first reviewer, and AC will be the second reviewer for the systematic review. SJ drafted the manuscript. All authors read and approved the final manuscript.

\section{Funding}

None.

Availability of data and materials

Not applicable.

Ethics approval and consent to participate

Not applicable.

Consent for publication

Not applicable.

\section{Competing interests}

The authors declare that they have no competing interests.

\section{Author details}

${ }^{1}$ Health Technology Assessment in India, Department of Health Research, Ministry of Health and Family Welfare, Indian Red Cross Society Building, 1, Red Cross Road, New Delhi 110001, India. ${ }^{2}$ Indian Council of Medical Research, (ICMR), New Delhi, India.

Received: 27 November 2018 Accepted: 22 July 2019

Published online: 13 August 2019

References

1. Ju H, Hewson K. Health technology assessment and evidence-based policy making: queensland department of health experience. Int J Technol Assess Health Care. 2014;30(06):595-600 Available from: http://www.journals. cambridge.org/abstract_S0266462314000695. [cited 2018 Jul 11].

2. Stevens A, Milne R. Health technology assessment in England and Wales. Int J Technol Assess Health Care. 2004;20(1):11-24 Available from: http://www. ncbi.nlm.nih.gov/pubmed/15176173. [cited 2018 Jul 11].

3. Pwee $\mathrm{K}$, Tong $\mathrm{S}$, Chow W. Health technology assessment supports evidence-based decision-making in the Changi general hospital marketplace exercise. Value Heal. 2016;19(7):A831-2 Available from: http:// linkinghub.elsevier.com/retrieve/pii/S109830151631155X. [cited 2018 Jul 11].

4. About us | Department of Health Research | MoHFW | Government of India. Available from: https://dhr.gov.in/about-mtab. [cited 2018 May 31].

5. Prinja S, Downey LE, Gauba VK, Swaminathan S. Health technology assessment for policy making in India: current scenario and way forward. Pharmacoecon Open 2018; 2(1):1-3. Available from: https://doi.org/10.1007/ s41669-017-0037-0. [cited 2018 May 31].

6. Downey LE, Mehndiratta A, Grover A, Gauba V, Sheikh K, Prinja S, et al. Institutionalising health technology assessment: establishing the Medical Technology Assessment Board in India. BMJ Glob Heal. 2017; 
2(2):e000259. Available from: https://doi.org/10.1136/bmjgh-2016000259. [cited 2018 May 31].

7. RSBY: Rashtriya Swasthya Bima Yojna. Available from: http://www.rsby.gov. in/. [cited 2017 Jul 21].

8. Ayushman Bharat -Pradhan Mantri Jan AarogyaYojana (AB-PMJAY) to be launched by Prime Minister Shri Narendra Modi in Ranchi, Jharkahnd on September 23, 2018. Available from: http://pib.nic.in/newsite/PrintRelease. aspx?relid=183624. [cited 2018 Sep 24].

9. Facts About Cataract | National Eye Institute [Internet]. [cited 2019 Jul 28]. Available from: https://nei.nih.gov/health/cataract/cataract_facts.

10. WHO | Cataract. WHO. 2015; Available from: http://www.who.int/topics/ cataract/en/. [cited 2017 Jul 21].

11. Thulasiraj RD, Rahamathulla R, Saraswati A, Selvaraj S, Ellwein LB. The Sivaganga eye survey: I. blindness and cataract surgery. Ophthalmic Epidemiol. 2002:9(5):299-312.

12. Thulasiraj RD, Nirmalan PK, Ramakrishnan R, Krishnadas R, Manimekalai TK, Baburajan NP, et al. Blindness and vision impairment in a rural South Indian population: the Aravind Comprehensive Eye Survey. Ophthalmology. 2003;110(8):1491-8.

13. Nirmalan PK, Thulasiraj RD, Maneksha V, Rahmathullah R, Ramakrishnan R, Padmavathi $A$, et al. A population based eye survey of older adults in Tirunelveli district of South India: blindness, cataract surgery, and visual outcomes. Br J Ophthalmol. 2002;86(5):505-12.

14. Murthy GV, Ellwein LB, Gupta S, Tanikachalam K, Ray M, Dada VK. A population-based eye survey of older adults in a rural district of Rajasthan: II. Outcomes of cataract surgery. Ophthalmology. 2001;108(4):686-92.

15. Limburg H, Kumar R. Follow-up study of blindness attributed to cataract in Karnataka State, India. Ophthalmic Epidemiol. 1998:5(4):211-23 Available from: http://www.ncbi.nlm.nih.gov/pubmed/9894805. [cited 2017 Jul 21].

16. Rajavi Z, Katibeh M, Ziaei H, Fardesmaeilpour N, Sehat M, Ahmadieh H, et al. Rapid assessment of avoidable blindness in Iran. Ophthalmology. 2011; 118(9):1812-1818. Available from: https://doi.org/10.1371/journal.pone.0002 867. Myer L, editor. [cited 2017 Jul 21].

17. Cataract Surgery - American Academy of Ophthalmology. Available from: https://www.aao.org/eye-health/diseases/what-is-cataract-surgery. [cited 2018 Nov 19].

18. WHO. Priority eye diseases: WHO; 2018. Available from: http://www.who.int/ blindness/causes/priority/en/index1.html. [cited 2018 Nov 19]

19. Porter RB. Global Initiative The Economic Case. Community Eye Heal. 1998; 11(27):44-5 Available from: http://www.ncbi.nlm.nih.gov/pubmed/17492041. [cited 2018 Nov 19].

20. Ellwood PM. Shattuck lecture--outcomes management. A technology of patient experience. 1988. Arch Pathol Lab Med. 1997;121(11):1137-44.

21. Speight J, Reaney MD, Barnard KD. Not all roads lead to Rome - a review of quality of life measurement in adults with diabetes. Diabet Med. 2009;26(4): 315-27 Available from: http://www.ncbi.nlm.nih.gov/pubmed/19388959. [cited 2017 Oct 24].

22. Brazier J, Muston D, Konwea H, Power GS, Barzey V, Lloyd A, et al. Evaluating the relationship between visual acuity and utilities in patients with diabetic macular edema enrolled in intravitreal aflibercept studies. Investig Ophthalmol Vis Sci. 2017;58(11):4818-25.

23. Jain $\mathrm{S}$, Trials CC. International prospective register of systematic reviews The effects of cataract surgery and intra-ocular lens implantation on vision function and quality of life in age related cataract patients : a systematic review Review question Participants / popula; 2018. p. 1-3.

24. Ara R, Brazier J, Peasgood T, Paisley S. The identification, review and synthesis of health state utility values from the literature. Pharmacoeconomics. 2017;35(Suppl 1):1-13.

\section{Publisher's Note}

Springer Nature remains neutral with regard to jurisdictional claims in published maps and institutional affiliations.

Ready to submit your research? Choose BMC and benefit from:

- fast, convenient online submission

- thorough peer review by experienced researchers in your field

- rapid publication on acceptance

- support for research data, including large and complex data types

- gold Open Access which fosters wider collaboration and increased citations

- maximum visibility for your research: over $100 \mathrm{M}$ website views per year

At BMC, research is always in progress.

Learn more biomedcentral.com/submissions 